\title{
Production and Chemical Composition of Extracellular Polysaccharides of Rhizobium
}

\author{
By SURESH K. GHAI, MAKOTO HISAMATSU, AKINORI AMEMURA \\ AND TOKUYA HARADA* \\ The Institute of Scientific and Industrial Research, Osaka University, Yamada-kami, Suita, \\ Osaka 565, Japan
}

(Received 29 April 1980; revised 8 July 1980)

\begin{abstract}
Five cultures of Rhizobium meliloti (J7017, 202, 204, 207, 209) and one of Rhizobium trifolii (J60) produced water-soluble polysaccharides containing glucose, galactose and pyruvic acid in a molar ratio of $7: 1: 1$ and some succinic and acetic acids. These were identified as succinoglycan-like polysaccharides on the basis of their components, methylation analysis and fragmentation with two specific $\beta$-glycanases. One culture of $R$. meliloti (IFO 13336) produced water-soluble polysaccharide containing glucose, galactose, glucuronic acid and acetic acid in a molar ratio of $5: 1: 1: 2$, and an unidentified component. Two cultures of $R$. meliloti $(201,206)$ produced water-soluble polysaccharides containing glucose, galactose, mannose and glucuronic acid in a molar ratio of $4: 2: 3: 1$ and $4: 1: 2: 1$, respectively, and some pyruvic acid. Rhizobium trifolii IFO 13337 and $R$. japonicum IFO 13338 produced water-soluble polysaccharides containing glucose, galactose, glucuronic acid, pyruvic acid and acetic acid in a molar ratio of $6: 1: 1: 2: 1$. Two isolates from the stock culture of $R$. trifolii $\mathrm{J} 60$ produced large amounts of the water-insoluble polysaccharide curdlan. This is the first report in Rhizobium of the occurrence of curdlan and of spontaneous mutation in ability to produce succinoglycan-like polysaccharide and curdlan.
\end{abstract}

\section{INTRODUCTION}

The extracellular polysaccharides of Rhizobium strains are of interest because they are thought to play a role in determining the specificity of the bacteria for their host legume plants. Various attempts have been made to relate the extracellular polysaccharide composition of Rhizobium strains to their host specificity. All strains of a given Rhizobium species, except $R$. japonicum, appear to produce similar polysaccharides (Dudman, 1976). Rhizobium trifolii, $R$. leguminosarum and $R$. phaseoli apparently produce identical poiysaccharides (Zevenhuizen, 1973), while the polysaccharides of $R$. meliloti are identical to those of Agrobacterium tumefaciens (Zevenhuizen, 1973) and Agrobacterium radiobacter (Hisamatsu et al., 1978). We thought it interesting to continue studies on the role of bacterial polysaccharides in interaction with the host plant because $A$. tumefaciens, which produces extracellular polysaccharide, causes crown gall tumours in plants.

The polysaccharide of one strain of $R$. meliloti (U27) was studied by Jansson et al. (1977). Comparative studies (Harada et al., 1979) showed that the water-soluble polysaccharides from $R$. meliloti U27, Alcaligenes faecalis var. myxogenes $10 \mathrm{C} 3$ and Agrobacterium radiobacter have the same structure, except for the ratio of their contents of the succinyl or acetyl radical: the sugar moiety is shown in formula $I$.

We now report the composition of the extracellular polysaccharides of a number of Rhizobium strains and the quantity of polysaccharide formed during growth. 


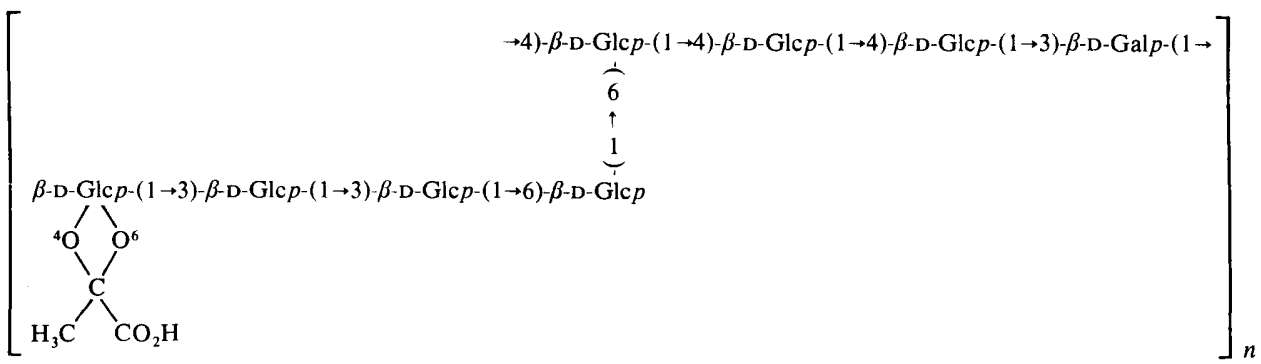

Formula I

\section{METHODS}

Strains. Rhizobium meliloti IFO 13336, R. trifolit IFO 13337 and $R$. japonicum IFO 13338 were obtained from the Institute for Fermentation, Osaka, Japan; $R$. meliloti J7017 and $R$. trifolii J60 were supplied by Professor Y. Maruyama, University of Tokyo, Japan; R. meliloti strains 201, 202, 204, 206, 207 and 209 were supplied by Professor M. Yatazawa, Nagoya University, Japan.

Media. Strains were maintained on slopes of yeast extract/mannitol agar medium (Graham, 1965). The medium used to examine polysaccharide production contained (per $100 \mathrm{ml}$ distilled water): glucose, $4 \mathrm{~g} ;\left(\mathrm{NH}_{4}\right)_{2} \mathrm{HPO}_{4}$, $0.15 \mathrm{~g} ; \mathrm{KH}_{2} \mathrm{PO}_{4}, 0.1 \mathrm{~g} ; \mathrm{MgSO}_{4} .7 \mathrm{H}_{2} \mathrm{O}, 0.05 \mathrm{~g} ; \mathrm{NaCl}, 1 \mathrm{mg} ; \mathrm{CaCl}_{2}, 1 \mathrm{mg} ; \mathrm{MnCl}_{2} .4 \mathrm{H}_{2} \mathrm{O}, 1 \mathrm{mg} ;$ thiamin, $20 \mu \mathrm{g}$; biotin, $2 \mu \mathrm{g} ; \mathrm{CaCO}_{3}, 0.5 \mathrm{~g} ; \mathrm{pH}$ 7.0. Aniline blue medium (Nakanishi et al., 1974), used to detect colonies forming curdlan, contained (per $100 \mathrm{ml}$ distilled water): glucose, $1 \mathrm{~g}$; yeast extract, $0.5 \mathrm{~g}$; aniline blue, $5 \mathrm{mg}$; agar, $1.5 \mathrm{~g}$; pH 7.0.

Culture conditions for polysaccharide production. The medium $(95 \mathrm{ml})$ in $500 \mathrm{ml}$ conical flasks was inoculated with a seed culture $(5 \mathrm{ml})$ which had been grown in the same medium. The culture was shaken reciprocally at 120 strokes $\min ^{-1}$ at $30^{\circ} \mathrm{C}$ for $5 \mathrm{~d}$.

Separation and analysis of precipitates (cells including cell surface polysaccharide). On centrifuging the culture broth, cells and $\mathrm{CaCO}_{3}$ were precipitated. The $\mathrm{CaCO}_{3}$ was removed by washing with $1 \mathrm{M}-\mathrm{HCl}$ and then the remaining precipitate was washed with water, dehydrated with acetone and weighed. The amount of sugars in the precipitate was assayed by the phenol $/ \mathrm{H}_{2} \mathrm{SO}_{4}$ method (Dubois et al., 1956) using glucose as a standard. Nitrogen in the precipitate was assayed with a CHN-Corder, type M72 (Yanagimoto, Tokyo, Japan), and the amount of protein was calculated by multiplying the value by $6 \cdot 25$.

Preparation of water-soluble polysaccharide. Water-soluble polysaccharide was prepared by the method described previously (Misaki et al., 1969) involving precipitation with acetone and cetylpyridinium chloride.

Quantitative analysis of sugars. Water-soluble polysaccharide was hydrolysed in a sealed tube with $1 \mathrm{ml} 90 \%$ formic acid for $16 \mathrm{~h}$ at $100^{\circ} \mathrm{C}$, and then with $0.5 \mathrm{ml} 4 \mathrm{M}$-trifluoroacetic acid for $6 \mathrm{~h}$ at $100^{\circ} \mathrm{C}$. The hydrolysate was converted to a mixture of alditol acetates and analysed (Björndal et al., 1967) in a CG7A gas chromatograph (Shimadzu, Tokyo, Japan) fitted with a fiame-ionization detector and a column $(4 \times 200 \mathrm{~mm})$ of $3 \%(w / w)$ ECNSS-M on Gas-chrom Q (Applied Science Laboratories) at $190^{\circ} \mathrm{C}$. Xylose was used as an internal standard in these analyses.

Uronic acids were determined by the modified carbazole reaction described by Galambos (1967).

Quantitative analysis of organic acids. Pyruvic acid in water-soluble polysaccharide was assayed by the method of Koepsell \& Sharpe (1952). Succinic acid and acetic acid were assayed by liquid chromatography as described previously (Hisamatsu et al., 1978).

Preparation of water-insoluble polysaccharide. The precipitate containing cells $(200 \mathrm{mg})$ was homogenized with $50 \mathrm{ml} 0.5 \mathrm{M}-\mathrm{NaOH}$ and centrifuged at $20000 \mathrm{rev} . \mathrm{min}^{-1}$ for $30 \mathrm{~min}$. The supernatant was neutralized with $1 \mathrm{M}-\mathrm{HCl}$ and the precipitate thus formed was collected by centrifugation, washed with water, dehydrated by addition of acetone and then dried in vacuo.

Successive digestion of polysaccharides with succinoglycan depolymerase and intracellular endo- $(1 \rightarrow 6)$ $\beta$-D-glucanase of Flavobacterium M64. A solution of $0.1 \%(w / v)$ polysaccharide was stirred in $10 \mathrm{mM}-\mathrm{KOH}$ for $5 \mathrm{~h}$ at $20^{\circ} \mathrm{C}$ under nitrogen to remove acyl groups. Deacylated polysaccharide $(1 \mathrm{mg})$ was dissolved in $200 \mu 1$ $0.1 \mathrm{M}$-sodium acetate buffer ( $\mathrm{pH} \mathrm{5.8)}$ ) and incubated with $100 \iota \mathrm{l}$ succinoglycan depolymerase ( 2 units) for $5 \mathrm{~h}$ at $40^{\circ} \mathrm{C}$. The mixture was then boiled for $1 \mathrm{~min}$, mixed with 100,1 intracellular endo-( $\left.1 \rightarrow 6\right)$ - $\beta$-D-glucanase $(2$ units $)$ and incubated for another $5 \mathrm{~h}$. The mixture was boiled again for $1 \mathrm{~min}$ and passed through a column $(10 \times 50 \mathrm{~mm})$ of Amberlite IR-120 $\left(\mathrm{H}^{+}\right.$resin). The effluent was evaporated in vacuo and subjected to paper chromatography to examine the hydrolysis products.

Preparation of succinoglycan depolymerase and intracellular endo- $(1 \rightarrow 6)-\beta$-D-glucanase. Succinoglycan depolymerase and intracellular endo- $(1 \rightarrow 6)-\beta$-D-glucanase were prepared as described previously (Amemura $e t$ al., 
1974; Abe et al., 1980). One unit of enzyme activity is defined as the amount of enzyme which liberates $1 \mu \mathrm{mol}$ aldehyde group as glucose $\mathrm{h}^{-1}$.

Paper chromatography. Samples were applied to Toyo-Roshi no. 50 paper (Toyo. Tokyo, Japan) and developed with a solvent system of 1-butanol/pyridine/water $\left(6: 4: 3\right.$, by vol.). Sugars were detected with $\mathrm{AgNO}_{3}$ reagent. The solvent used to separate uronic acids was 1-butanol/acetic acid/water (4:1:5, by vol.).

Methylation analysis. Samples $(2 \mathrm{mg}$ ) were methylated and analysed as described previously (Hisamatsu et al., 1980).

\section{RESULTS AND DISCUSSION}

\section{Isolation of colonies on aniline blue plates}

Stock cultures of $R$. meliloti strains IFO 13336, J7017, 201, 202, 204, 206, 207 and 209, $R$. trifolii strains IFO 13337 and $\mathrm{J} 60$ and $R$. japonicum strain IFO 13338 were suspended in saline and spread on aniline blue plates. After $5 \mathrm{~d}$ at $30^{\circ} \mathrm{C}$, colonies of all the strains were slimy. Rhizobium meliloti strains 201 and 206 and $R$. trifolii IFO 13337 formed white colonies which stained slightly with the dye, while other strains formed unstained colonies.

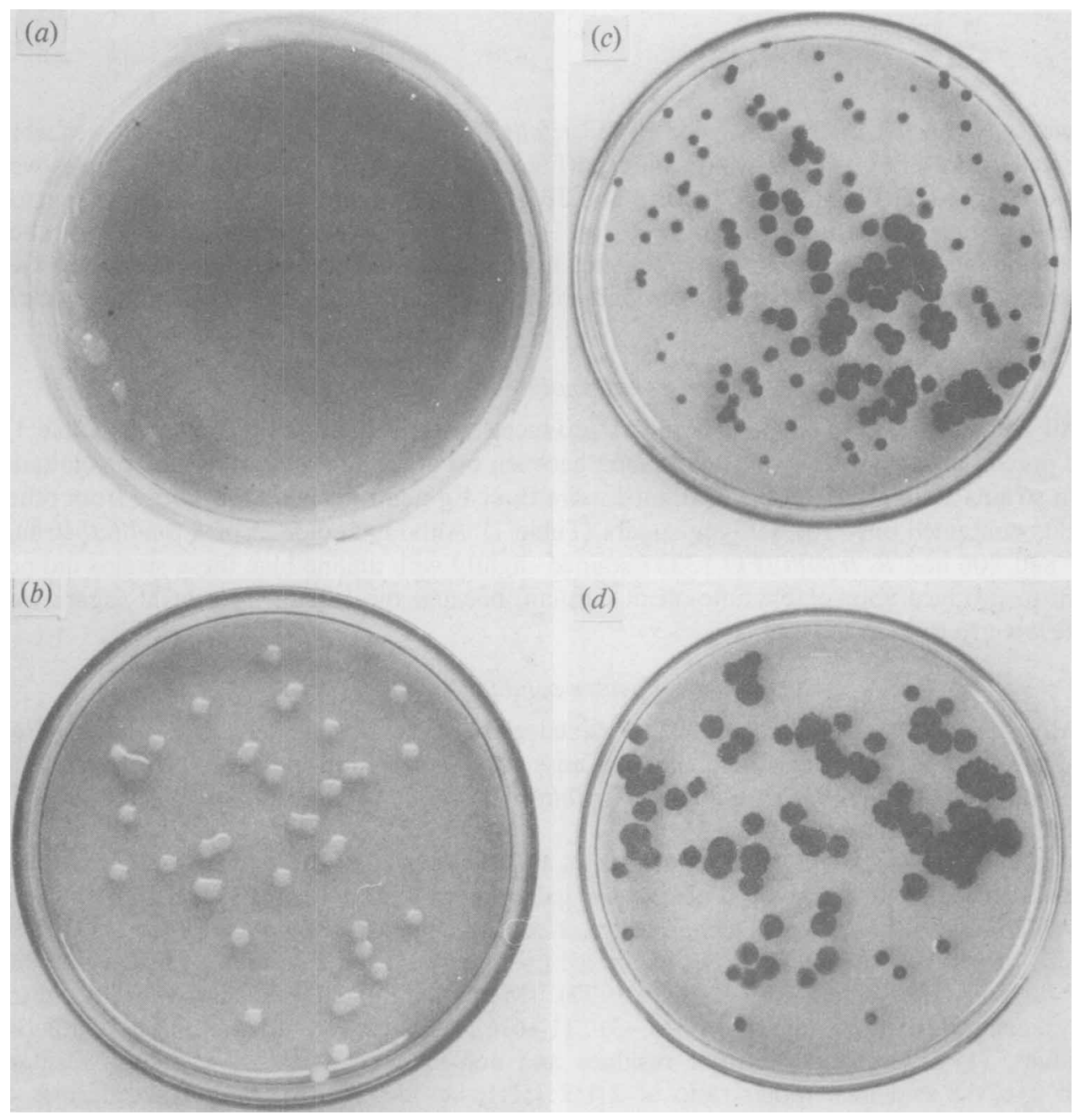

Fig. 1. Colonies of Rhizobium trifolii J60 after growth at $30^{\circ} \mathrm{C}$ for $5 \mathrm{~d}$ on aniline blue medium: (a) stock culture; (b) strain J60W; (c) strain J60B1; (d) strain J60B2. 
Table 1. Polysaccharides produced by Rhizobium strains

\begin{tabular}{|c|c|c|c|c|}
\hline \multirow[b]{2}{*}{ Strain } & \multirow{2}{*}{$\begin{array}{l}\text { Water-soluble } \\
\text { polysaccharide } \\
{\left[\mathrm{mg}(100 \mathrm{ml})^{-1}\right]}\end{array}$} & \multicolumn{3}{|c|}{ Precipitate $\left[\mathrm{mg}(100 \mathrm{ml})^{-1}\right]$} \\
\hline & & Total wt & Protein & Carbohydrate \\
\hline \multicolumn{5}{|l|}{ R. meliloti } \\
\hline IFO 13336 & 840 & 500 & 140 & 90 \\
\hline J7017 & 220 & 200 & 126 & 15 \\
\hline 201 & 255 & 93 & 58 & 11 \\
\hline 202 & 330 & 450 & 198 & 30 \\
\hline 204 & 355 & 630 & 221 & 43 \\
\hline 206 & 450 & 140 & 72 & 11 \\
\hline 207 & 340 & 730 & 204 & 50 \\
\hline 209 & 92 & 270 & 179 & 44 \\
\hline \multicolumn{5}{|l|}{ R. trifolii } \\
\hline IFO 13337 & 150 & 280 & 78 & 10 \\
\hline J60W & 580 & 310 & 162 & 35 \\
\hline $\mathrm{J} 60 \mathrm{~B} 1$ & 0 & 1460 & 210 & 1180 \\
\hline $\mathrm{J} 60 \mathrm{~B} 2$ & 18 & 1430 & 200 & 1240 \\
\hline \multicolumn{5}{|l|}{$R$. japonicum } \\
\hline IFO 13338 & 600 & 420 & 184 & 11 \\
\hline
\end{tabular}

However, when the thick suspension of $R$. trifolii $\mathrm{J} 60$ was plated, many blue colonies were detected in the slimy lawn of white colonies (Fig. 1a); the ratio of blue to white colonies was $1: 380$. White $(\mathrm{J} 60 \mathrm{~W})$ and blue (J60B 1 and $\mathrm{J} 60 \mathrm{~B} 2)$ colony types were purified by repeated single colony isolation for further study (Fig. 1). We have previously reported the appearance on aniline blue plates of similar blue colonies from stock cultures of Alcaligenes faecalis var. myxogenes and several strains of Agrobacterium (Amemura et al., 1977; Hisamatsu et al., 1977).

\section{Production of water-soluble polysaccharides and precipitates}

All the strains except $R$. trifolii J60B1 produced water-soluble polysaccharide (Table 1). The precipitates from all strains contained between 60 and $220 \mathrm{mg}$ protein. The precipitates from strains J60B1 and J60B2 contained more than $1 \mathrm{~g}$ neutral sugars, but those from other strains contained only 10 to $90 \mathrm{mg}$ sugars (Table 1 ). Although colonies of $R$. meliloti strains 201 and 206 and $R$. trifolii IFO 13337 stained slightly with aniline blue these strains did not seem to produce appreciable amount of curdlan, because the content of neutral sugars was quite low (10 to $11 \mathrm{mg})$.

\section{Components of water-soluble polysaccharides}

All water-soluble polysaccharides contained glucose and galactose; those of $R$. meliloti strains 201 and 206 also contained mannose, and the soluble polysaccharides from $R$. meliloti IFO 13336, 201 and 206, $R$. trifolii IFO 13337 and $R$. japonicum IFO 13338 contained glucuronic acid (Table 2).

The polysaccharides of $R$. meliloti strains J7017, 202, 204, 207 and 209 and $R$. trifolii J60W had the same molar ratio of glucose, galactose and pyruvic acid (7:1:1). This ratio is the same as that of the polysaccharides of Alcaligenes faecalis var. myxogenes 10C 3 , nine strains of Agrobacterium which we examined previously (Hisamatsu et al., 1978, 1980) and R. meliloti U27 (Jansson et al., 1977). Methylation analysis showed that these polysaccharides were composed of $(1 \rightarrow 3)$-, $(1 \rightarrow 6)-,(1 \rightarrow 4)$ - and $(1 \rightarrow 4,1 \rightarrow 6)$-linked D-glucose residues, $(1 \rightarrow 3)$-linked $\mathrm{D}$-galactose residues and non-reducing terminal $\mathrm{D}$-glucose residues with pyruvic acid in a molar ratio of $2: 1: 2: 1: 1: 1$, i.e. identical to the polysaccharides of Alcaligenes faecalis var. myxogenes 10C3, Agrobacterium radiobacter, Agrobacterium tumefaciens, Agrobacterium rhizogenes and R. meliloti U27 (Hisamatsu et al., 1980).

The polysaccharide of $R$. meliloti IFO 13336 contained glucose, galactose and glucuronic 


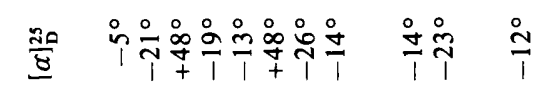

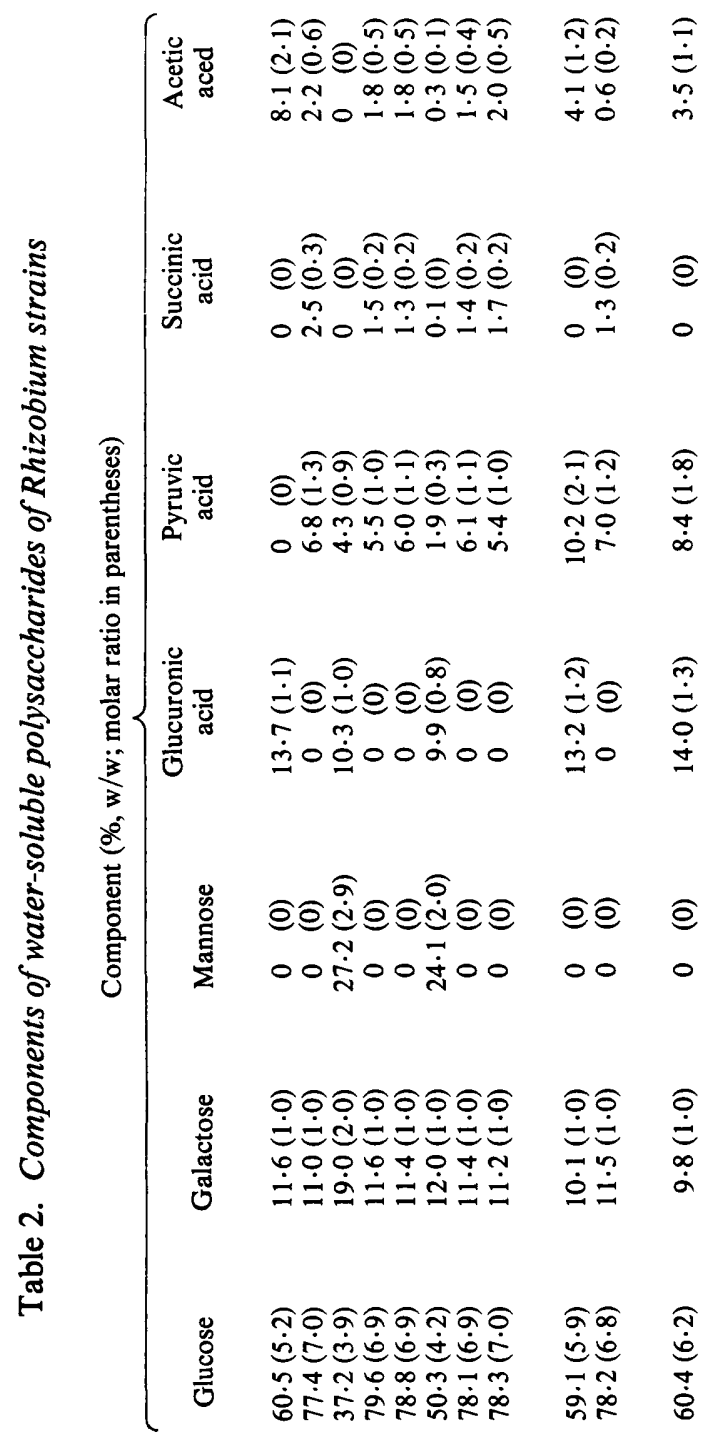

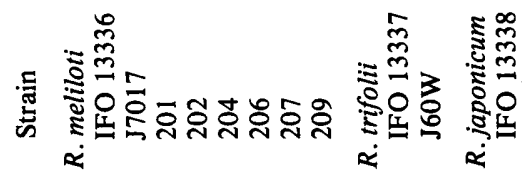


acid in a molar ratio of $5: 1: 1$, but no pyruvic acid. The polysaccharides of $R$. meliloti strains 201 and 206 contained mannose and their molar ratios of glucose, galactose, mannose and glucuronic acid were $4: 2: 3: 1$ and $4: 1: 2: 1$, respectively. These polysaccharides also contained some pyruvic acid. Rhizobium trifolii IFO 13337 and $R$. japonicum IFO 13338 produced similar polysaccharides with molar ratios of glucose, galactose, glucuronic acid and pyruvic acid of $6: 1: 1: 2$. This ratio is similar to that reported for polysaccharides of other strains of $R$. trifolii (Chaudhari et al., 1973; Sømme, 1974: Jansson \& Lindberg, 1979).

All the polysaccharides except that of strain 201 contained succinic acid and/or acetic acid. An unidentified acidic substance that was released by treatment with weak acid was present in the polysaccharide of $R$. meliloti IFO 13336.

Dudman $(1976,1978)$ reported compositional heterogeneity in the polysaccharides of $R$. japonicum. Our results indicate heterogeneity in the polysaccharides of $R$. meliloti and $R$. trifolii.

Digestion of water-soluble polysaccharides with succinoglycan depolymerase and intracellular endo- $(1 \rightarrow 6)-\beta$-D-glucanase of Flavobacterium

Previously (Hisamatsu et al., 1978; Harada et al., 1979; Abe et al., 1980) we reported that water-soluble polysaccharides (deacylated) from Alcaligenes faecalis var. myxogenes 10C3, nine strains of Agrobacterium and $R$. meliloti U27 were hydrolysed by successive treatment with succinoglycan depolymerase and intracellular endo- $(1 \rightarrow 6)-\beta$-D-glucanase to produce two oligosaccharides. By analysing these oligosaccharides we showed that the polysaccharides have the same structure irrespective of their modes of acylation (Hisamatsu et al., 1980). Since these enzymes have high specificity (Amemura et al., 1974; Abe et al., 1980) we tested whether they could hydrolyse the polysaccharides of the various Rhizobium strains. The polysaccharides of $R$. meliloti strains J7017, 202, 204, 207 and 209 and $R$. trifolii J60W were hydrolysed by succinoglycan depolymerase, as was the polysaccharide (succinoglycan) of Alcaligenes faecalis var. myxogenes, to give a single spot of reducing material near the origin of the paper chromatogram. Moreover, these polysaccharides were hydrolysed successively by succinoglycan depolymerase and intracellular $(1 \rightarrow 6)-\beta$-D-glucanase to give two spots of oligosaccharides which had the same $R_{F}$ values as those from succinoglycan $\left(R_{F} 0.27\right.$ and $0 \cdot 11$ ).

Rhizobium meliloti IFO 13336 polysaccharide was hydrolysed by succinoglycan depolymerase (the activity was about $5 \%$ of that for succinoglycan) and was also hydrolysed by the intracellular enzyme of Flavobacterium, but the pattern of the final products on the paper chromatogram was different $\left(R_{F} 0 \cdot 11\right.$ and 0$)$.

No other polysaccharides were hydrolysed by these enzymes. These results show that the polysaccharides of $R$. meliloti strains J7017, 202, 204, 207 and 209 and $R$. trifolii J60W may have substantially the same structure as succinoglycan. The polysaccharide of $R$. meliloti IFO 13336 has a different structure, but it also is susceptible to the enzymes, probably because it contains the components $\rightarrow 3)$-Gal- $(1 \rightarrow 4)$-Glc- $(1 \rightarrow$ and $\rightarrow 6)$-Glc- $(1 \rightarrow 6)$-Glc- $(1 \rightarrow$.

\section{Water-insoluble polysaccharide of R. trifolii $J 60$}

A sample of $580 \mathrm{mg}$ of the water-insoluble polysaccharide of $R$. trifolii J60B 1 was obtained from $1 \mathrm{~g}$ of the precipitate by extraction with $0.5 \mathrm{M}-\mathrm{NaOH}$. The polysaccharide stained with aniline blue, like curdlan from Alcaligenes faecalis var. myxogenes and strains of Agrobacterium. The optical rotation $[\alpha]_{\mathrm{D}}^{20}$ was $+12^{\circ}(c 1$ in $1 \mathrm{M}-\mathrm{NaOH})$. After complete hydrolysis of the polysaccharide only glucose was detected by paper chromatography, while after partial hydrolysis glucose, laminaribiose, laminaritriose and higher laminari-oligosaccharides were detected. When a $2 \%(\mathrm{w} / \mathrm{v})$ suspension of the polysaccharide in water was heated at $90^{\circ} \mathrm{C}$ for $10 \mathrm{~min}$, it made a firm gel with a strength of $740 \mathrm{~g} \mathrm{~cm}^{-2}$, like that of curdlan from Alcaligenes faecalis var. myxogenes 10C3K (Maeda et al., 1967). Methylation 
analysis established that 2,4,6-tri- $O$-methyl-D-glucose was the predominant product of hydrolysis of the fully methylated polysaccharide. From these results it was concluded that the water-insoluble polysaccharide was curdlan. Curdlan has not previously been shown to be present in Rhizobium strains.

Strain J60W mutated spontaneously to make curdlan during prolonged storage ( 2 months) on yeast extract/mannitol agar slants, as has previously been reported for Alcaligenes faecalis var. myxogenes (Amemura et al., 1977) and several strains of Agrobacterium (Hisamatsu et al., 1977). Rhizobium trifolii J60 produces succinoglycan-like polysaccharide as well as curdlan, like Alcaligenes faecalis var. myxogenes (Harada, 1965, 1977; Harada et al., $1968 a, b$ ) and Agrobacterium strains (Nakanishi et al., 1976; Hisamatsu et al., 1978). It is interesting that spontaneous mutation in the ability to produce these types of polysaccharides occurs in a strain of Rhizobium as well as Alcaligenes faecalis var. myxogenes and Agrobacterium strains.

We thank Professor Y. Maruyama, University of Tokyo, and Professor M. Yatazawa, Nagoya University, for gifts of Rhizobium strains.

\section{REFERENCES}

Abe, J., Amemura, A. \& Harada, T. (1980). An endo-(1 $\rightarrow 6)-\beta$-D-glucanase of Flavobacterium M64 hydrolyzing the octasaccharide repeating unit of succinoglycan to two tetrasaccharides. Agricultural and Biological Chemistry 44, 1877-1884.

Amemura, A., Moori, K. \& Harada, T. (1974). Purification and properties of a specific, inducible $\boldsymbol{\beta}$-glucanase, succinoglucan depolymerase from Flavobacterium. Biochimica et biophysica acta 334, 398-409.

Amemura, A., Hisamatsu, M. \& Harada, T. (1977). Spontaneous mutation of polysaccharide production in Alcaligenes faecalis var. myxogenes 10C3. Applied and Environmental Microbiology 34, 617-620.

BJöRndAL, H., LiNDBERG, B. \& SvensSON, S. (1967). Gas-liquid chromatography of partially methylated alditols as their acetates. Acta chemica scandinavica 21, 1801-1804.

Chaudhari, A. S., Bishop, C. T. \& Dudman, W. F. (1973). Structural studies on the specific capsular polysaccharide from Rhizobium trifolii TA-1. Carbohydrate Research 28, 221-231.

Dubois, M., Gilles, K. A., Hamilton, J. K., Rebers, P. A. \& SMITH, F. (1956). Colorimetric method for determination of sugars and related substances. Analytical Chemistry 28, 350-356.

Dudman, W. (1976). The extracellular polysaccharides of Rhizobium japonicum: compositional studies. Carbohydrate Research 46, 97-110.

Dudman, W. F. (1978). Structural studies of the extracellular polysaccharides of Rhizobium japonicum strains 71A, CC708 and CB1795. Carbohydrate Research 66, 9-23.

GaLAmBOS, J. T. (1967). The reaction of carbazole with carbohydrates. 1 . Effect of borate and sulfamate on the carbazole color of sugars. Analytical Biochemistry 19, 119-132.

GraHAM, P. H. (1965). Extracellular polysaccharides of the genus Rhizobium. Antonie van Leeuwenhoek 31, 349-354.

Harada, T. (1965). Succinoglucan 10C3: a new polysaccharide of Alcaligenes faecalis var. myxogenes. Archives of Biochemistry and Biophysics 112, 65-69.

HaRADA, T. (1977). Production, properties, and application of curdlan. ACS Symposium Series 45, 265-283.

Harada, T., Amemura, A., Saito, H., Kanamaru, S. \& MISAKI, A. (1968a). Formation of succinoglucan and curdlan by parent and mutant strains of Alcaligenes faecalis var. myxogenes 10C3. Journal of Fermentation Technology 46, 679-684.

Harada, T., Misaki, A. \& SAITo, H. (1968b). Curdlan: a bacterial gel-forming $\beta$-1,3-glucan. Archives of Biochemistry and Biophysics 124, 292-298.

Harada, T., Amemura, A., Jansson, P.-E. \& LINDBERG, B. (1979). Comparative studies of polysaccharides elaborated by Rhizobium, Alcaligenes and Agrobacterium. Carbohydrate Research 77, 285-288.

Hisamatsu, M., Ott, I., Amemura, A. \& Harada, T. (1977). Change in ability of Agrobacterium to produce water-soluble and water-insoluble $\beta$ glucans. Journal of General Microbiology 103, 375-379.

Hisamatsu, M., Sano, K., Amemura, A. \& Harada, T. (1978). Acidic polysaccharides containing succinic acid in various strains of Agrobacterium. Carbohydrate Research 61, 89-96.

Hisamatsu, M., Abe, J., Amemura, A. \& Harada, T. (1980). Structural elucidation on succinoglycan and related polysaccharides from Agrobacterium and Rhizobium by fragmentation with two special $\beta$-D-glycanases and methylation analysis. Agricultural and Biological Chemistry 44, 1049-1055.

JANSSON, P.-E. \& LindBERG, B. (1979). Structural studies of the Rhizobium trifolii extracellular polysaccharide. Carbohydrate Research 75, 207-220.

Jansson, P.-E. KenNe, L., LindBerG, B., LJungGREN, H., LönNGRen, J., RudÉn, V. \& SvensSon, A. (1977). Demonstration of an octasaccharide repeating unit in the extracellular polysaccharide of Rhizobium meliloti by sequential degradation. Journal of the American Chemical Society 99, 3812-3815. 
Koepsell, H. J. \& Sharpe, E. S. (1952). Microdetermination of pyruvic acid and $a$-ketoglutaric acids. Archives of Biochemistry and Biophysics 38, 443-449.

Maeda, I., Sarto, H., Masada, M., Misaki, A. \& HARADA, T. (1967). Properties of gels formed by heat treatment of curdlan, a bacterial $\beta$-1,3-glucan. Agricultural and Biological Chemistry 31, 1184 1188.

Misaki, A., SaIto, H., Ito, T. \& Harada, T. (1969). Structure of succinoglucan, and extracellular acidic polysaccharide of Alcaligenes faecalis var. myxogenes. Biochemistry 8, 4645-4650.

NaKanishi, I., Kimura, K., Kusui, S. \& Yamazaki, E. (1974). Complex formation of gel-forming bacterial $(1 \rightarrow 3)-\beta$-D-glucan (curdlan-type polysac- charides) with dyes in aqueous solution. Carbohydrate Research 32, 47-52.

NaKanishi, I., Kimura, K., Suzuki, T., IshikaWa, M., Banno, I., Sakane, T. \& Harada, T. (1976). Demonstration of curdlan-type polysaccharide and some other $\beta$-1,3-glucan in micro-organisms with aniline blue. Journal of General and Applied Microbiology 22, 1-11.

Søмme, R. (1974). Chemical analysis of exocellular, acidic polysaccharides from seven Rhizobium strains. Carbohydrate Research 33, 89-96.

Zevenhuizen, L. P. T. M. (1973). Methylation analysis of acidic exopolysaccharides of Rhizobium and Agrobacterium. Carbohydrate Research 26, 409-419. 